\title{
TH2A-SS-2
}

\section{Topical 5-aminolevulinic acid photodynamic therapy for the treatment of warts: comparison of red and green light-emitting diode array}

\author{
Hsiung-Fei Chien ${ }^{a}$, MD. Ph.D., Yi-Hua Liao ${ }^{b}$, MD. , Chin-Tin Chen ${ }^{\mathrm{c}}$, Ph.D. \\ a. Department of Surgery \\ b: Department of Dermatology \\ : Center for Optoelectronic Biomedicine \\ College of Medicine, National Taiwan University \\ Taipei 100 , Taiwan \\ e-mail: hfchien@ha.mc.ntu.edu.tw; ctchen@ha.mc.ntu.edu.tw
}

\begin{abstract}
The purpose of this clinical study is to develop topical ALA-PDT as an alternative treatment of warts but without the unwanted side effects of pain and burning. Topical ALA-PDT with green LED array proved to be an effective and painless treatment modality for warts.
\end{abstract}

Verruca vulgaris are benign skin papillomas caused by the human papilloma virus (HPV). They are very common and can affect many different sites including the face, hands, feet and genitalia. Although the present therapeutic approaches are more or less effective in eradicating the lesions, relapses are very common.

Furthermore, at times the anatomical location and depth of lesions often make the treatment difficult, time-consuming and painful. Therefore, it is necessary to develop new modalities for wart treatment [1-2].

Previously, using the homemade LED light source, we have shown that ALA-PDT is effective for the treatment of premalignant lesions such as mucosal dysplasia and carcinoma in situ of oral cavity. Due to the advantages of low cost, high reliability, and portability, LED light source provides an alternative approach for the light irradiation of PDT.

The major characteristics in favor of LED used for PDT are price and versatility. LED is inexpensive (in comparison with all the other sources described so far) and can be arranged in array to irradiate large areas. Moreover, they can be arranged in different geometric combination to compensate for difficult anatomic areas. To choose a light source for PDT, reliability, ease of use, cost and space are the most important variables that need to be considered in a clinical setting. We have previously shown that topical ALA application together with a convenient and easy-to-use LED light source is an attractive development for the treatment of oral dysplasia. In addition, ALA PDT using LED light source is also effective in the killing of bacterial in 
suspension culture and biofilm. These results clearly indicate the possibility of using ALA PDT combined with LED light source for the infectious disease, such as warts. We have previously test the efficacy of ALA-PDT on a patient with wart at the finger of right hand using Green LED array under the light dose of $15 \mathrm{~J} / \mathrm{cm}^{2}$ ( light intensity: $25 \mathrm{~mW} / \mathrm{cm}^{2}$ ). During the treatment, patient experienced only a slight pain in the last minute of light irradiation (treatment time: 10 minutes) and no anaesthesia was used. Clinical photographs before and after treatment is shown in Figure 1. Following ALA-PDT, a moderate swelling and erythema of the irradiated area was observed, but no uncomfortable pain was reported as shown in Figure 1C. The complete response was found 3 weeks post ALA-PDT and no reoccurrence was observed up to 10 months.

In this study, we address the possibility of combining topical ALA and LED array for the photodynamic therapy of warts. To fulfill this goal, an ALA formulation for topical application was developed to limit the ALA penetration at the wart lesions. To reduce the severe pain and burning, green light as well as red light LED array will be developed and used to compare the treatment efficacy and possible side effects of ALA-PDT during the treatment of warts.

\section{References}

[1] Stender I-M, Lock-Andersen J and Wulf HC. Recalcitrant hand and foot warts successful treated with photodynamic therapy with topical 5-aminolaevulinic acid: a pilot study. Clin Exp Dermatol 24:154-159, 1999.

[2] Stender I-M, Na R, Fogh $\mathrm{H}$ et al., Photodynamic therapy with 5-aminolaevulinic acid or placebo for recalcitrant hand and foot warts: randomized double-blind trial.

Lancet 355:963-966, 2000.

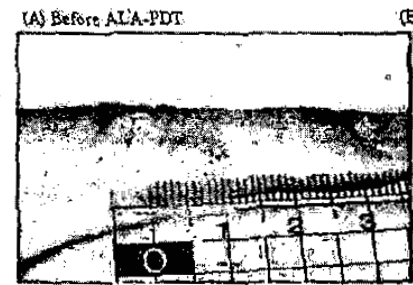

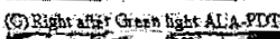

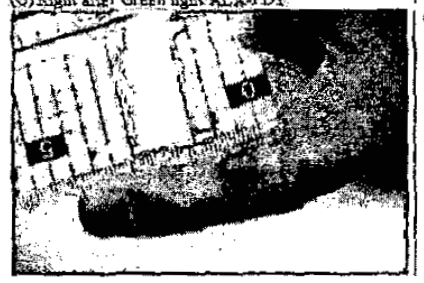

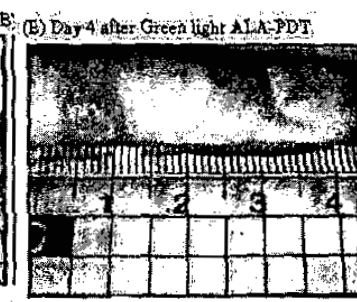

(G) Day 18 afte Greti light AI'A-PDT

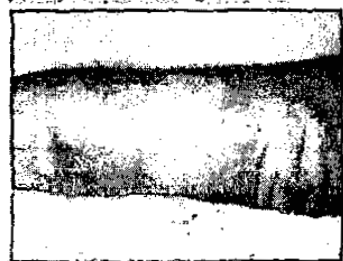

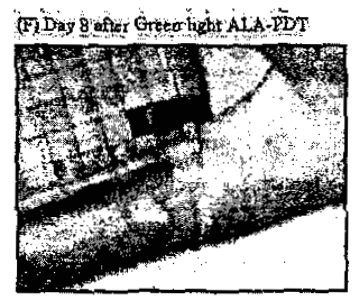

(F) 10 atoto followedis aftorALA-PDT

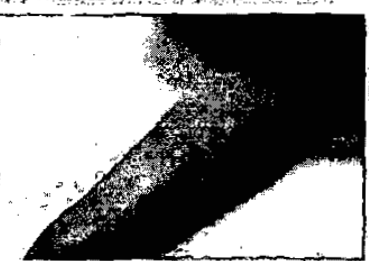

Figure 1: Preliminary study of complete responding wart on the finger before and after ALA-PDT. 